\title{
Synthesis and anti-tumor evaluation of novel hydrazide and hydrazide-hydrazone derivatives
}

\author{
WAGNAT WAHBA WARDAKHAN ${ }^{1, *}$ \\ NAHED NASSER EID EL-SAYED ${ }^{1,2}$ \\ RAFAT MILAD MOHAREB 3 \\ ${ }^{1}$ National Organization for Drug Control \\ and Research P.O. 29 Cairo, A. R. Egypt \\ ${ }^{2}$ College of Science, Department \\ of Chemistry, King Saud University \\ Women Students - Medical Studies \\ and Science Sections \\ P.O. Box 22452, Riyadh 11495 \\ Kingdom of Saudi Arabia \\ ${ }^{3}$ Department of Chemistry \\ Faculty of Science, Cairo University \\ Giza, A. R. Egypt
}

Accepted September 28, 2012
The reaction of cyclopentanone with cyanoacetylhydrazine gave 2-cyano-2-cyclopentylideneacetohydrazide (1). Treatment of compound $\mathbf{1}$ with elemental sulphur in the presence of triethylamine afforded 2-amino-5,6-dihydro-4H-cyclopenta[ $b]$ thiophene-3-carbohydrazide (2), which in-turn formed the corresponding intermediate diazonium salt. The latter was coupled with either ethyl cyanoacetate or ethyl acetoacetate to form 2-cyano-2-(3-(hydrazinecarbonyl)-5,6-dihydro-4H-cyclopenta[ $b]$ thiophen-2-yl)hydrazono)acetate (3) and ethyl 2-(2-(3-(hydrazinecarbonyl)-5,6-dihydro-4H-cyclopenta[ $b$ ] thiophen-2-yl)hydrazono)-3-oxobutanoate (4), respectively. On the other hand, the reaction of compound 1 with either benzaldehyde or acetophenone afforded $N^{\prime}$-benzylidene-2-cyano-2-cyclopentylideneacetohydrazide (7) and 2-cyano-2-(2-cyclopentylidene)phenylacetohydrazide (10), respectively. Moreover, compound 1 was used to synthesize 2-cyano-2-cyclopentylidene- $N$ '-(arylthiazol-2(3H)-ylidene)acetohydrazides $(6 \mathbf{a}, \mathbf{b}), 2$-(2-benzylidenecyclopentylidene)-2-cyanoacetohydrazide (8), 2-amino- $N$ '-benzylidene-5,6-dihydro- $4 H$ -cyclopenta[b]thiophene-3-carbohydrazide (9), 2-cyano-2-(2-(2-phenylhydrazono)cyclopentylidene)acetohydrazide (11), $N^{\prime}$-(1-chloropropan-2-ylidene)-2-cyano-2-cyclopentylideneacetohydrazide (12), and 2-cyclopentylidene-3-(3,5-disubstituted-1H-pyrazol-1-yl)-3-oxopropanenitriles $(\mathbf{1 3 a}, \mathbf{b})$ through its reaction with the respective reagents. Antitumor evaluation of the newly synthesized compounds against the three human tumor cells lines, namely, breast adenocarcinoma (MCF-7), non-small cell lung cancer (NCI-H460) and CNS cancer (SF-268) showed that some of the described compounds exhibited higher inhibitory effects towards the three tumor cell lines than the reference compound doxorubicin.

Keywords: hydrazide, hydrazide-hydrazone, thiophene, pyrazole, antitumor

\footnotetext{
* Correspondence; e-mail: wagnatward@hotmail.com
} 
In many reports, hydrazide-hydrazones are considered to be good candidates for different pharmaceutical applications, where such compounds were considered as antibacterial, antifugal, antimicrobial and anticonvulsant agents (1-8). Moreover, many of them showed analgesic and antiplatelet properties. Therapeutic prominence of the hidrazide-hydrazone derivatives has been well established. In addition, hydrazide-hydrazones were reported to elicit anticancer (13-20) and antiHIV properties (21) and hence they have gained an important place in medicinal chemistry. Recently, hydrazide-hydrazones have gained great importance due to their diverse biological properties, including anti-inflammatory, antimalarial and antituberculotic activities (22-27). With the aim of obtaining novel hydrazide-hydrazones with a wide spectrum of pharmaceutical applications, we report herein the synthesis of a series of hydrazide-hydrazones and some of their heterocyclic transformations, followed by antitumor evaluations of newly synthesized products $(28,29)$.

\section{EXPERIMENTAL}

All melting points were determined on an electrothermal apparatus (Büchi 535, Switzerland) in an open capillary tube and are uncorrected. Elemental analyses were performed on a Yanaco CHNS Corder elemental analyzer (Japan). IR spectra $\left(v, \mathrm{~cm}^{-1}\right)$ were recorded in $\mathrm{KBr}$ pellets on a PA-9721 IR spectrophotometer (Shimadzu, Japan). ${ }^{1} \mathrm{H}$ $\mathrm{NMR}$ and ${ }^{13} \mathrm{C}$ NMR spectra were obtained on a Jeol $300 \mathrm{MHz}$ (Japan) spectrometer in DMSO- $d_{6}$ as a solvent, using TMS as internal reference and chemical shifts $(\delta)$ are expressed in ppm. Mass spectra were recorded on Kratos (75 eV) MS equipment (Germany). Synthetic pathways are presented in Schemes 1-3 and physicochemical and spectral data of the synthesized compounds are given in Tables I and II.

\section{Syntheses}

2-Cyano-2-cyclopentylideneacetohydrazide (1). - To a solution of cyanoacetylhydrazine $(1.00 \mathrm{~g}, 0.01 \mathrm{~mol})$ in 1 ,4-dioxane $(30 \mathrm{~mL})$ containing piperidine $(0.50 \mathrm{~mL})$, cyclopentanone $(0.84 \mathrm{~g}, 0.01 \mathrm{~mol})$ was added. The reaction mixture was heated under reflux for $2 \mathrm{~h}$ and then left to cool. The solid product formed upon pouring onto ice/water containing a few drops of hydrochloric acid was collected by filtration.

2-Amino-5,6-dihydro-4H-cyclopenta[b]thiophene-3-carbohydrazide (2). - A mixture of compound 1 (1.65 g, $0.01 \mathrm{~mol})$ and elemental sulphur $(0.32 \mathrm{~g}, 0.01 \mathrm{~mol})$ in absolute ethanol $(30 \mathrm{~mL})$ containing triethylamine $(0.80 \mathrm{~mL})$ was heated under reflux for $2 \mathrm{~h}$. The solid product formed upon pouring onto ice/water containing a few drops of hydrochloric acid was collected by filtration.

Ethyl 2-cyano-2-(3-(hydrazinecarbonyl)-5,6-dihydro-4H-cyclopenta[b]thiophen-2-yl)hydrazono)acetate (3) and ethyl 2-(2-(3-(hydrazinecarbonyl)-5,6-dihydro-4H-cyclopenta[b]thiophen-2-yl)hydrazono)-3-oxobutanoate (4). General procedure. - A cold solution $\left(0-5{ }^{\circ} \mathrm{C}\right)$ of 1 (1.65 g, $0.01 \mathrm{~mol})$ in acetic acid $(10 \mathrm{~mL})$ was added to a cold solution of nitrosyl sulphuric acid [prepared by the addition of sodium nitrite solution $(0.7 \mathrm{~g}, 0.01 \mathrm{~mol})$ to a cold solution of concentrated sulphuric acid $(8 \mathrm{~mL})$, then the resulting mixture was heated at $60{ }^{\circ} \mathrm{C}$ for $1 \mathrm{~h}$ and then cooled to $0{ }^{\circ} \mathrm{C}$ ] under continuous stirring to form the appropriate diazonium sul- 
W. W. Wardakhan et al.: Synthesis and anti-tumor evaluation of novel hydrazide and hydrazide-hydrazone derivatives, Acta Pharm. 63 (2013) 45-57.

Table I. Analytical data of compounds 1-13

\begin{tabular}{|c|c|c|c|c|c|c|c|}
\hline \multirow[t]{2}{*}{ Compd. } & \multirow{2}{*}{$\begin{array}{c}\text { M.p. }\left({ }^{\circ} \mathrm{C}\right) \\
\text { (solvent of cryst.) }\end{array}$} & \multirow{2}{*}{$\begin{array}{l}\text { Yield } \\
(\%)\end{array}$} & \multirow{2}{*}{$\begin{array}{l}\text { Mol. formula } \\
\qquad\left(M_{\mathrm{r}}\right)\end{array}$} & \multicolumn{4}{|c|}{ Analysis (calcd./found, \%) } \\
\hline & & & & C & $\mathrm{H}$ & $\mathrm{N}$ & $S$ \\
\hline 1 & $\begin{array}{c}>300 \\
(\mathrm{EtOH})\end{array}$ & 60 & $\begin{array}{c}\mathrm{C}_{8} \mathrm{H}_{11} \mathrm{~N}_{3} \mathrm{O} \\
(165.19)\end{array}$ & $\begin{array}{l}58.17 \\
58.24\end{array}$ & $\begin{array}{l}6.71 \\
6.49\end{array}$ & $\begin{array}{l}25.44 \\
25.29\end{array}$ & \\
\hline 2 & $\begin{array}{l}280-283 \\
(\mathrm{EtOH})\end{array}$ & 77 & $\begin{array}{l}\mathrm{C}_{8} \mathrm{H}_{11} \mathrm{~N}_{3} \mathrm{OS} \\
(197.26)\end{array}$ & $\begin{array}{l}48.71 \\
48.92\end{array}$ & $\begin{array}{l}5.62 \\
5.49\end{array}$ & $\begin{array}{l}21.30 \\
21.49\end{array}$ & $\begin{array}{l}16.26 \\
16.45\end{array}$ \\
\hline 3 & $\begin{array}{l}198-201 \\
(\mathrm{EtOH})\end{array}$ & 80 & $\begin{array}{c}\mathrm{C}_{13} \mathrm{H}_{15} \mathrm{~N}_{5} \mathrm{O}_{3} \mathrm{~S} \\
(321.35)\end{array}$ & $\begin{array}{l}48.59 \\
48.77\end{array}$ & $\begin{array}{l}4.70 \\
4.90\end{array}$ & $\begin{array}{l}21.79 \\
21.62\end{array}$ & $\begin{array}{l}9.98 \\
10.1\end{array}$ \\
\hline 4 & $\begin{array}{l}222-225 \\
(\mathrm{EtOH})\end{array}$ & 68 & $\begin{array}{c}\mathrm{C}_{14} \mathrm{H}_{18} \mathrm{~N}_{4} \mathrm{O}_{4} \mathrm{~S} \\
(338.38)\end{array}$ & $\begin{array}{l}49.69 \\
49.81\end{array}$ & $\begin{array}{l}5.36 \\
5.02\end{array}$ & $\begin{array}{l}16.56 \\
16.82\end{array}$ & $\begin{array}{l}9.48 \\
9.55\end{array}$ \\
\hline 5 & $\begin{array}{c}160-162 \\
\text { (1,4-dioxane) }\end{array}$ & 73 & $\begin{array}{l}\mathrm{C}_{15} \mathrm{H}_{16} \mathrm{~N}_{4} \mathrm{OS} \\
(300.38)\end{array}$ & $\begin{array}{l}59.98 \\
60.27\end{array}$ & $\begin{array}{l}5.37 \\
5.29\end{array}$ & $\begin{array}{l}18.65 \\
18.30\end{array}$ & $\begin{array}{l}10.67 \\
10.82\end{array}$ \\
\hline $6 a$ & $\begin{array}{c}158-160 \\
\text { (1,4-dioxane) }\end{array}$ & 70 & $\begin{array}{l}\mathrm{C}_{18} \mathrm{H}_{18} \mathrm{~N}_{4} \mathrm{OS} \\
(338.43)\end{array}$ & $\begin{array}{l}63.88 \\
64.07\end{array}$ & $\begin{array}{l}5.36 \\
5.51\end{array}$ & $\begin{array}{l}16.56 \\
16.73\end{array}$ & $\begin{array}{l}9.47 \\
9.33\end{array}$ \\
\hline $6 b$ & $\begin{array}{l}192-195 \\
(\mathrm{EtOH})\end{array}$ & 80 & $\begin{array}{l}\mathrm{C}_{23} \mathrm{H}_{20} \mathrm{~N}_{4} \mathrm{OS} \\
(400.50)\end{array}$ & $\begin{array}{l}68.98 \\
68.72\end{array}$ & $\begin{array}{l}5.03 \\
5.31\end{array}$ & $\begin{array}{l}13.99 \\
13.85\end{array}$ & $\begin{array}{l}8.01 \\
8.22\end{array}$ \\
\hline 7 & $\begin{array}{l}282-284 \\
(\mathrm{EtOH})\end{array}$ & 69 & $\begin{array}{c}\mathrm{C}_{15} \mathrm{H}_{15} \mathrm{~N}_{3} \mathrm{O} \\
(253.30)\end{array}$ & $\begin{array}{l}71.13 \\
71.14\end{array}$ & $\begin{array}{l}5.97 \\
5.77\end{array}$ & $\begin{array}{l}16.59 \\
16.82\end{array}$ & \\
\hline 8 & $\begin{array}{l}208-210 \\
(\mathrm{EtOH})\end{array}$ & 75 & $\begin{array}{c}\mathrm{C}_{15} \mathrm{H}_{15} \mathrm{~N}_{3} \mathrm{O} \\
(253.30)\end{array}$ & $\begin{array}{l}71.13 \\
71.17\end{array}$ & $\begin{array}{l}5.97 \\
5.84\end{array}$ & $\begin{array}{l}16.59 \\
16.39\end{array}$ & \\
\hline 9 & $\begin{array}{l}266-270 \\
(\mathrm{EtOH})\end{array}$ & 86 & $\begin{array}{c}\mathrm{C}_{15} \mathrm{H}_{15} \mathrm{~N}_{3} \mathrm{OS} \\
(285.36)\end{array}$ & $\begin{array}{l}63.13 \\
63.09\end{array}$ & $\begin{array}{l}5.30 \\
5.51\end{array}$ & $\begin{array}{l}14.73 \\
14.51\end{array}$ & $\begin{array}{l}11.24 \\
11.09\end{array}$ \\
\hline 10 & $\begin{array}{l}140-143 \\
(\mathrm{EtOH})\end{array}$ & 88 & $\begin{array}{c}\mathrm{C}_{16} \mathrm{H}_{17} \mathrm{~N}_{3} \mathrm{O} \\
(267.33)\end{array}$ & $\begin{array}{l}71.89 \\
71.94\end{array}$ & $\begin{array}{l}6.41 \\
6.27\end{array}$ & $\begin{array}{l}15.72 \\
16.01\end{array}$ & \\
\hline 11 & $\begin{array}{c}166-168 \\
\text { (1,4-dioxane) }\end{array}$ & 90 & $\begin{array}{c}\mathrm{C}_{14} \mathrm{H}_{15} \mathrm{~N}_{5} \mathrm{O} \\
(269.30)\end{array}$ & $\begin{array}{l}62.44 \\
62.61\end{array}$ & $\begin{array}{l}5.61 \\
5.72\end{array}$ & $\begin{array}{l}26.01 \\
25.82\end{array}$ & \\
\hline 12 & $\begin{array}{l}122-125 \\
(\mathrm{EtOH})\end{array}$ & 75 & $\begin{array}{c}\mathrm{C}_{11} \mathrm{H}_{14} \mathrm{ClN}_{3} \\
\mathrm{O}(239.70)\end{array}$ & $\begin{array}{l}55.12 \\
55.09\end{array}$ & $\begin{array}{l}5.89 \\
6.03\end{array}$ & $\begin{array}{l}17.53 \\
17.72\end{array}$ & \\
\hline $13 a$ & $\begin{array}{c}210-212 \\
\text { (1,4-dioxane) }\end{array}$ & 66 & $\begin{array}{c}\mathrm{C}_{11} \mathrm{H}_{13} \mathrm{~N}_{5} \mathrm{O} \\
(231.25)\end{array}$ & $\begin{array}{l}57.28 \\
57.38\end{array}$ & $\begin{array}{l}5.89 \\
5.55\end{array}$ & $\begin{array}{l}30.41 \\
30.22\end{array}$ & \\
\hline $13 b$ & $\begin{array}{c}270-273 \\
\text { (1,4-dioxane) }\end{array}$ & 57 & $\begin{array}{c}\mathrm{C}_{11} \mathrm{H}_{12} \mathrm{~N}_{4} \mathrm{O}_{2} \\
(232.24)\end{array}$ & $\begin{array}{l}56.89 \\
56.72\end{array}$ & $\begin{array}{l}5.21 \\
5.47\end{array}$ & $\begin{array}{l}24.12 \\
24.06\end{array}$ & \\
\hline
\end{tabular}

phate salt as yellow precipitate. The formed cold solution of the diazonium salt was added to a cold solution of either ethyl cyanoacetate $(1.13 \mathrm{~g}, 0.01 \mathrm{~mol})$ or ethyl acetoacetate $(1.0 \mathrm{~g}, 0.01 \mathrm{~mol})$ in ethanol $(95 \%, 40 \mathrm{~mL})$ containing sodium acetate $(4.0 \mathrm{~g})$ under continuous stirring. The formed solid product, in each case, was collected by filtration.

2-(2-Cyano-2-cyclopentylideneacetyl)-N-phenylhydrazinecarbothioamide (5). - To a solution of compound $1(1.65 \mathrm{~g}, 0.01 \mathrm{~mol})$ in absolute ethanol $(40 \mathrm{~mL})$ containing triethylamine $(0.5 \mathrm{~mL})$, phenylisothiocyanate $(1.35 \mathrm{~g}, 9.91 \mathrm{~mol})$ was added. The reaction mixture was heated under reflux in a boiling water bath for $3 \mathrm{~h}$ and then poured onto ice/water. The formed solid product was collected by filtration. 
W. W. Wardakhan et al.: Synthesis and anti-tumor evaluation of novel hydrazide and hydrazide-hydrazone derivatives, Acta Pharm. 63 (2013) $45-57$.

Table II. Spectral data of the newly synthesized compounds

\begin{tabular}{|c|c|c|c|c|}
\hline Compd. & $\operatorname{IR}\left(v, \mathrm{~cm}^{-1}\right)$ & $\begin{array}{l}{ }^{1} \mathrm{H} \text { NMR } \\
\left(\mathrm{DMSO}-d_{6}\right) \\
(\delta, \mathrm{ppm})\end{array}$ & $\begin{array}{l}{ }^{13} \mathrm{C} \text { NMR } \\
\left(\mathrm{DMSO}-d_{6}\right) \\
(\delta, \mathrm{ppm})\end{array}$ & $\begin{array}{c}\mathrm{MS} \\
\left(\mathrm{M}^{+}, m / z\right)\end{array}$ \\
\hline 1 & $\begin{array}{l}3325-3212\left(\mathrm{NH}_{2}\right. \\
\mathrm{NH}), 2885\left(\mathrm{CH}_{2}\right) \\
2257(\mathrm{CN}), 1678 \\
(\mathrm{CO}), 1647(\mathrm{C}=\mathrm{C})\end{array}$ & $\begin{array}{l}1.17-2.34(2 \mathrm{~m}, 8 \mathrm{H}, \\
\left.4 \mathrm{CH}_{2}\right), 3.83(\mathrm{~s}, 2 \mathrm{H}, \\
\left.\mathrm{NH}_{2}\right), 8.54(\mathrm{~s}, 1 \mathrm{H}, \\
\mathrm{NH})\end{array}$ & $\begin{array}{l}\text { 28.5, 28.7, 39.4, } 165.0 \text { (cyclopentyl } \\
\mathrm{C}), 90.3(\mathrm{C}=\mathrm{Cq}-\mathrm{CN}), 115.8(\mathrm{CN}) \\
165.8(\mathrm{C}=\mathrm{O})\end{array}$ & 165 \\
\hline 2 & $\begin{array}{l}3456-3322\left(2 \mathrm{NH}_{2}\right. \\
\mathrm{NH}), 2893\left(\mathrm{CH}_{2}\right) \\
1686(\mathrm{CO}), 1648 \\
(\mathrm{C}=\mathrm{C})\end{array}$ & $\begin{array}{l}1.16-2.37(2 \mathrm{~m}, 6 \mathrm{H}, \\
\left.3 \mathrm{CH}_{2}\right), 3.80,4.66 \\
\left(2 \mathrm{~s}, 4 \mathrm{H}, \mathrm{D}_{2} \mathrm{O}\right. \\
\text { exchangeable, } \\
\left.2 \mathrm{NH}_{2}\right), 8.77(\mathrm{~s}, 1 \mathrm{H}, \\
\mathrm{D}_{2} \mathrm{O} \text { exchangeable, } \\
\mathrm{NH})\end{array}$ & $\begin{array}{l}\text { 22.0, 28.5, } 32.8 \text { (cyclopentyl C), } \\
118.3,133.6,139.4,145.2 \text { (thiophene } \\
\text { C), } 164.9(\mathrm{C}=\mathrm{O})\end{array}$ & 197 \\
\hline 3 & $\begin{array}{l}3477-3320\left(\mathrm{NH}_{2}\right. \\
2 \mathrm{NH}), 2920,2890 \\
\left(\mathrm{CH}_{3}, \mathrm{CH}_{2}\right), 2253 \\
(\mathrm{CN}), 1720(\mathrm{CO}) \\
1678(\mathrm{CO}), 1644 \\
(\mathrm{C}=\mathrm{C}), 1630(\mathrm{C}=\mathrm{N})\end{array}$ & $\begin{array}{l}1.13(\mathrm{t}, 3 \mathrm{H}, J=7.02 \\
\left.\mathrm{Hz}, \mathrm{CH}_{3}\right), 1.15-2.35 \\
\left(2 \mathrm{~m}, 6 \mathrm{H}, 3 \mathrm{CH}_{2}\right) \\
3.87\left(\mathrm{~s}, 2 \mathrm{H}, \mathrm{NH}_{2}\right) \\
4.22(\mathrm{q}, 2 \mathrm{H}, J=7.02 \\
\left.\mathrm{Hz}, \mathrm{CH}_{2}\right), 8.81, \\
10.27(2 \mathrm{~s}, 2 \mathrm{H}, 2 \mathrm{NH})\end{array}$ & $\begin{array}{l}16.8\left(\mathrm{CH}_{3}\right), 22.0,28.5,(\text { cyclopentyl } \\
\mathrm{C}), 59.5\left(\mathrm{CH}_{2}\right), 116.3(\mathrm{CN}), 118.0 \\
133.7,139.2,145.6(\text { thiophene } \mathrm{C}) \\
162.8,166.9(2 \mathrm{C}=\mathrm{O}), 171.3(\mathrm{C}=\mathrm{N})\end{array}$ & 321 \\
\hline 4 & $\begin{array}{l}3462-3327\left(\mathrm{NH}_{2}\right. \\
2 \mathrm{NH}), 2977,2895 \\
\left(\mathrm{CH}_{3}, \mathrm{CH}_{2}\right), 1700, \\
1683.1690(3 \mathrm{CO}) \\
1641(\mathrm{C}=\mathrm{C}), 1628 \\
(\mathrm{C}=\mathrm{N})\end{array}$ & $\begin{array}{l}1.14(\mathrm{t}, 3 \mathrm{H}, J=6.98 \\
\left.\mathrm{Hz}, \mathrm{CH}_{3}\right), 1.17-2.32 \\
\left(2 \mathrm{~m}, 6 \mathrm{H}, 3 \mathrm{CH}_{2}\right), \\
2.73\left(\mathrm{~s}, 3 \mathrm{H}, \mathrm{CH}_{3}\right), \\
3.87\left(\mathrm{~s}, 2 \mathrm{H}, \mathrm{NH}_{2}\right), \\
4.22(\mathrm{q}, 2 \mathrm{H}, \mathrm{J}= \\
\left.6.98 \mathrm{~Hz}, \mathrm{CH}_{2}\right), 8.74, \\
10.16(2 \mathrm{~s}, 2 \mathrm{H}, 2 \mathrm{NH})\end{array}$ & $\begin{array}{l}16.9\left(\mathrm{CH}_{3}\right), 22.4,26.4,28.2,29.3 \\
\left(\text { cyclopentyl } \mathrm{C} \& \mathrm{CH}_{3}-\mathrm{CO}\right), 59.8 \\
\left(\mathrm{CH}_{2}\right), 118.4,133.9,139.0,145.1 \\
\text { (thiophene } \mathrm{C}), 162.2,164.7,166.7(3 \\
\mathrm{C}=\mathrm{O}), 170.9(\mathrm{C}=\mathrm{N})\end{array}$ & 338 \\
\hline 5 & $\begin{array}{l}3452-3330(2 \mathrm{NH}) \\
3120(\mathrm{CH} \text { ar. }), 2893 \\
\left(\mathrm{CH}_{2}\right), 2252(\mathrm{CN}) \\
1690(\mathrm{C}=\mathrm{O}), 1632 \\
(\mathrm{C}=\mathrm{C}), 1335(\mathrm{C}=\mathrm{S})\end{array}$ & $\begin{array}{l}1.14-2.36(2 \mathrm{~m}, 8 \mathrm{H}, \\
\left.4 \mathrm{CH}_{2}\right), 7.28-7.34 \\
\left(\mathrm{~m}, 5 \mathrm{H}, \mathrm{C}_{6} \mathrm{H}_{5}\right) \\
8.82,9.11,10.09(3 \mathrm{~s}, \\
3 \mathrm{H}, 3 \mathrm{NH})\end{array}$ & $\begin{array}{l}28.8(2 \mathrm{C}), 29.6,32.0,164.9 \\
\text { (cyclopentyl C), 96.6 (C=Cq }-\mathrm{CN}) \text {, } \\
116.4(\mathrm{CN}), 122.8,124.0,126.8(2 \\
\mathrm{C}), 128.0,136.8(\text { Phenyl } \mathrm{C}), 162.0 \\
(\mathrm{C}=\mathrm{O}), 177.8(\mathrm{C}=\mathrm{S})\end{array}$ & 300 \\
\hline $6 a$ & $\begin{array}{l}3485-3325(\mathrm{NH}), \\
3062(\mathrm{CH} \text { aromatic }), \\
2980,2986\left(\mathrm{CH}_{3}\right. \\
\left.\mathrm{CH}_{2}\right), 2255(\mathrm{CN}) \\
1685(\mathrm{C}=\mathrm{O}), 1640 \\
(\mathrm{C}=\mathrm{C})\end{array}$ & $\begin{array}{l}1.18-2.37(2 \mathrm{~m}, 8 \mathrm{H}, \\
\left.4 \mathrm{CH}_{2}\right), 2.68(\mathrm{~s}, 3 \mathrm{H}, \\
\left.\mathrm{CH}_{3}\right), 7.22-7.41(\mathrm{~m}, \\
6 \mathrm{H}_{1} \mathrm{C}_{6} \mathrm{H}_{5} \text {, thiazole } \\
\mathrm{H}-5), 8.77(\mathrm{~s}, 1 \mathrm{H}, \\
\mathrm{NH})\end{array}$ & $\begin{array}{l}18.3\left(\mathrm{CH}_{3}\right)_{\prime \prime}(2 \text { carbons }) 29.3,32.2, \\
164.4(\text { cyclopentyl } \mathrm{C}), 96.1 \\
(\mathrm{C}=\mathrm{Cq}-\mathrm{CN}), 116.9(\mathrm{CN}), 121.9, \\
124.0,126.4,127.8,136.3 \text { (Phenyl } \\
\mathrm{C}), 99.8,137.9,148.3,(\text { thiazole } \mathrm{C}), \\
164.8(\mathrm{C}=\mathrm{O})\end{array}$ & 339 \\
\hline $6 b$ & $\begin{array}{l}3460-3329(\mathrm{NH}), \\
3198(\mathrm{CH} \text { aromatic }), \\
2978\left(\mathrm{CH}_{2}\right), 2250 \\
(\mathrm{CN}), 1687(\mathrm{CO}) \\
1638(\mathrm{C}=\mathrm{C})\end{array}$ & $\begin{array}{l}1.18-2.37(2 \mathrm{~m}, 8 \mathrm{H}, \\
\left.4 \mathrm{CH}_{2}\right), 7.28-7.39 \\
\left(\mathrm{~m}, 11 \mathrm{H}, 2 \mathrm{C}_{6} \mathrm{H}_{5}\right. \\
\text { thiazole } \mathrm{H}-4), 8.88 \\
(\mathrm{~s}, 1 \mathrm{H}, \mathrm{NH})\end{array}$ & $\begin{array}{l}28.2(2 \mathrm{C}), 29.0,38.5,164.1 \\
\left.\text { (cyclopentyl C), 95.9 (C=C } \mathrm{C}_{\mathrm{q}}-\mathrm{CN}\right), \\
115.6(\mathrm{CN}), 120.3(2 \mathrm{C}), 120.8(2 \mathrm{C}), \\
121.3,124.4,125.9,127.8,132.0 \\
\text { (two phenyl C), 99.2, 135.7, 143.7, } \\
\text { (thiazole C), } 165.2(\mathrm{C}=\mathrm{O})\end{array}$ & 400 \\
\hline
\end{tabular}


3477-3436 (NH)

$1.16-2.35(2 \mathrm{~m}, 8 \mathrm{H}, \quad 28.1(2 \mathrm{C}), 28.7,39.4,165.2$

$3193(\mathrm{CH}$ aromatic $\left.), 4 \mathrm{CH}_{2}\right), 6.88(\mathrm{~s}, 1 \mathrm{H}, \quad$ (cyclopentyl $\mathrm{C}), 90.6\left(\mathrm{C}=\mathrm{C}_{\mathrm{q}}-\mathrm{CN}\right)$,

$\left.2968\left(\mathrm{CH}_{2}\right), 2254 \quad \mathrm{CH}=\mathrm{N}\right), 7.30-7.38 \quad 115.9(\mathrm{CN}), 119.3(2 \mathrm{C}), 122.6(2 \mathrm{C})$,

$(\mathrm{CN}), 1689(\mathrm{CO}), \quad\left(\mathrm{m}, 5 \mathrm{H}, \mathrm{C}_{6} \mathrm{H}_{5}\right), 8.83$ 129.0, 133.8 (phenyl C), 165.8

$1631(\mathrm{C}=\mathrm{C})$

$(\mathrm{s}, 1 \mathrm{H}, \mathrm{NH})$

$(\mathrm{C}=\mathrm{O}), 170.3(\mathrm{C}=\mathrm{N})$

$8 \quad 3481-3330\left(\mathrm{NH}_{2}\right.$,

$\mathrm{NH}), 3198(\mathrm{CH}$

$1.18-2.32(2 \mathrm{~m}, 6 \mathrm{H}$

$28.0,28.9,36.2,160.2,165.8$

253

aromatic), 2970

$\left.3 \mathrm{CH}_{2}\right), 4.82(\mathrm{~s}, 2 \mathrm{H}$,

$\left.\mathrm{NH}_{2}\right), 6.87(\mathrm{~s}, 1 \mathrm{H}$,

$\left(\mathrm{CH}_{2}\right), 2250(\mathrm{CN})$,

$\mathrm{CH}=\mathrm{C}), 7.29-7.35$

(cyclopentyl C), $90.8(\mathrm{C}=\mathrm{Cq}-\mathrm{CN})$,

$116.4(\mathrm{CN}), 119.6,124.2,(2 \mathrm{C}) 128.7$

1692 (CO), 1635

$(\mathrm{C}=\mathrm{C})$

$\left(\mathrm{m}, 5 \mathrm{H}, \mathrm{C}_{6} \mathrm{H}_{5}\right), 8.80(\mathrm{C}=\mathrm{O}) ; 170.3(\mathrm{C}=\mathrm{N})$

(s, $1 \mathrm{H}, \mathrm{NH})$

9

3383-3342 (NH \&

$\left.\mathrm{NH}_{2}\right), 3170(\mathrm{CH}$

aromatic), 2978

$\left(\mathrm{CH}_{2}\right), 1685(\mathrm{CO})$,

$1636(\mathrm{C}=\mathrm{C})$

1.14-2.38 (2m, 6H, 22.2, 28.5, 32.4 (cyclo-pentyl C),

$\left.3 \mathrm{CH}_{2}\right), 5.32(\mathrm{~s}, 2 \mathrm{H}, \quad 117.9,120.3,122.7(2 \mathrm{C}), 126.9(2 \mathrm{C})$,

197

$\left.\mathrm{NH}_{2}\right), 6.80(\mathrm{~s}, 1 \mathrm{H}, \quad 130.1,132.8,139.4,147.3$ (phenyl,

$\mathrm{CH}=\mathrm{N}), 7.27-7.42 \quad$ thiophene $\mathrm{C}), 165.3(\mathrm{C}=\mathrm{O}), 172.4$

$\left(\mathrm{m}, 5 \mathrm{H}, \mathrm{C}_{6} \mathrm{H}_{5}\right), 8.88 \quad(\mathrm{C}=\mathrm{N})$

(s, $1 \mathrm{H}, \mathrm{NH})$

10

3465-3329 (NH), 3060

(CH aromatic), 2979,

$2880\left(\mathrm{CH}_{3}, \mathrm{CH}_{2}\right)$,

$2255(\mathrm{CN}), 1689$

(CO), $1632(\mathrm{C}=\mathrm{C})$

11 3489-3342 $\left(\mathrm{NH}_{2}, 2\right.$

$\mathrm{NH}), 2969\left(\mathrm{CH}_{2}\right)$,

$2229(\mathrm{CN}), 1688$

(CO), $1628(\mathrm{C}=\mathrm{C})$

1.16-2.37 $(2 \mathrm{~m}, 8 \mathrm{H}$,

$20.3\left(\mathrm{CH}_{3}\right), 26.8(2 \mathrm{C}), 28.2,39.8$,

165.0 (cyclopentyl C), 90.6

$\left.\mathrm{CH}_{3}\right), 7.34-7.41(\mathrm{~m}$,

$(\mathrm{C}=\mathrm{Cq}-\mathrm{CN}), 116.3(\mathrm{CN}), 120.3(2 \mathrm{C})$,

$\left.5 \mathrm{H}, \mathrm{C}_{6} \mathrm{H}_{5}\right), 8.37(\mathrm{~s}, \quad 122.7(2 \mathrm{C}), 128.9,132.9$ (phenyl $\mathrm{C}$ ), $1 \mathrm{H}, \mathrm{NH})$

$165.4(\mathrm{C}=\mathrm{O}), 170.8 \mathrm{C}=\mathrm{N})$

1.14-2.30 (2m, 6H, 26.9, 28.9, 30.1, 144.0, 165.9

$\left.3 \mathrm{CH}_{2}\right), 4.79(\mathrm{~s}, 2 \mathrm{H}$, (cyclopentyl C), $90.6(\mathrm{C}=\mathrm{Cq}-\mathrm{CN})$,

$\left.\mathrm{NH}_{2}\right), 7.26-7.39(\mathrm{~m}, 117.3(\mathrm{CN}), 120.3(2 \mathrm{C}), 122.7,128.9$

$\left.5 \mathrm{H}, \mathrm{C}_{6} \mathrm{H}_{5}\right), 8.32, \quad(2 \mathrm{C}), 131.8$ (phenyl $\left.\mathrm{C}\right), 165.9(\mathrm{C}=\mathrm{O})$

$8.79(2 \mathrm{~s}, 2 \mathrm{H}, 2 \mathrm{NH})$

$12 \quad 3449-3330(\mathrm{NH})$,

2981, $2893\left(\mathrm{CH}_{3}\right.$,

$\left.\mathrm{CH}_{2}\right), 2529(\mathrm{CN})$,

1692 (CO), 1629

$(\mathrm{C}=\mathrm{C})$

$1.15-2.39(2 \mathrm{~m}, 8 \mathrm{H}$,

$19.8\left(\mathrm{CH}_{3}\right), 26.9,28.3,28.2,33.0$,

$\left.4 \mathrm{CH}_{2}\right), 2.89\left(\mathrm{~s}, 3 \mathrm{H}, 165.0\right.$ (cyclopentyl C), $39.8\left(\mathrm{CH}_{2}\right)$,

$\left.\mathrm{CH}_{3}\right), 3.91(\mathrm{~s}, 2 \mathrm{H}$,

$\left.\mathrm{CH}_{2}\right), 8.63(\mathrm{~s}, 1 \mathrm{H}$,

$90.4(\mathrm{C}=\mathrm{Cq}-\mathrm{CN}), 116.8(\mathrm{CN}), 160.3$

$(\mathrm{C}=\mathrm{N}), \operatorname{165.4}(\mathrm{C}=\mathrm{O})$ $\mathrm{NH})$

13a $3449-3330\left(2 \mathrm{NH}_{2}\right)$, $2984\left(\mathrm{CH}_{2}\right), 2255$

(CN), $1698(\mathrm{CO})$,

$1626(\mathrm{C}=\mathrm{C})$

1.16-2.42 (2m, 8H,

$26.8,28.2,28.5,39.2,164.8$

(cyclopentyl C), 90.4 (C=Cq-CN),

$\left.4 \mathrm{CH}_{2}\right), 3.64,4.86$

$\left(2 \mathrm{~s}, 4 \mathrm{H}, 2 \mathrm{NH}_{2}\right)$,

$6.99(\mathrm{~s}, 1 \mathrm{H}$,

99.6, 146.9, 147.2 (pyrazole $C$ ),

$116.5(\mathrm{CN}), 166.3(\mathrm{C}=\mathrm{O})$

pyrazole $\mathrm{H}-4)$

13b 3520-3328 $(\mathrm{OH}$

$\left.\mathrm{NH}_{2}\right), 2981\left(\mathrm{CH}_{2}\right)$,

$2259(\mathrm{CN}), 1695$

1.17-2.38 $(2 \mathrm{~m}, 8 \mathrm{H}$,

$\left.4 \mathrm{CH}_{2}\right), 4.26(\mathrm{~s}, 2 \mathrm{H}$,

$\left.\mathrm{NH}_{2}\right), 6.89$ (s, $1 \mathrm{H}$,

(CO), $1629(\mathrm{C}=\mathrm{C})$

pyrazole $\mathrm{H}-4)$,

$10.22(\mathrm{~s}, 1 \mathrm{H}, \mathrm{OH})$

26.6, 28.0, 28.7, 39.5, 165.9

(cyclopentyl C), $91.0(\mathrm{C}=\mathrm{Cq}-\mathrm{CN})$,

$116.8(\mathrm{CN}), 99.3,134.8,158.6$

(pyrazole $\mathrm{C}), 166.0(\mathrm{C}=\mathrm{O})$

2-Cyano-2-cyclopentylidene-N'-(4-methyl-3-phenylthiazol-2(3H)-ylidene)acetohydrazide $\quad(6 a)$ and 2-cyano-2-cyclopentylidene- $\mathrm{N}^{\prime}-(3,4-d i p h e n y l t h i a z o l-2(3 \mathrm{H})$-ylidene)acetohydrazide (6b). General procedure. - To a solution of compound $5(3.00 \mathrm{~g}, 0.01 \mathrm{~mol})$ in absolute ethanol $(40 \mathrm{~mL})$, either $\alpha$-chloroacetone $(0.93 \mathrm{~g}, 0.8 \mathrm{~mL}, 0.01 \mathrm{~mol})$ or phenacyl bromide $(2.00 \mathrm{~g}, 0.01 \mathrm{~mol})$ 
was added. The reaction mixture was heated under reflux for $2 \mathrm{~h}$ and then poured onto water containing a few drops of sodium hydroxide solution $(0.25 \mathrm{~mL}, 10 \%)$. The formed solid product, in each case, was collected by filtration.

N'-Benzylidene-2-cyano-2-cyclopentylideneacetohydrazide (7). - To a solution of comound $1(1.65 \mathrm{~g}, 0.01 \mathrm{~mol})$ in 1,4-dioxane $(40 \mathrm{~mL})$, benzaldehyde $(1.06 \mathrm{~g}, 0.01 \mathrm{~mol})$ was added. The reaction mixture was heated under reflux for $2 \mathrm{~h}$, then poured onto ice/water containing a few drops of hydrochloric acid and the formed solid product was collected by filtration.

2-(2-Benzylidenecyclopentylidene)-2-cyanoacetohydrazide (8). - To a solution of compound $1(1.65 \mathrm{~g}, 0.01 \mathrm{~mol})$ in 1,4-dioxane $(40 \mathrm{~mL})$ containing piperidine $(0.5 \mathrm{~mL})$, benzaldehyde $(1.06 \mathrm{~g}, 0.01 \mathrm{~mol})$ was added. The reaction mixture was heated under reflux for $2 \mathrm{~h}$, then poured onto ice/water containing a few drops of hydrochloric acid and the formed solid product was collected by filtration.

2-Amino-N'-benzylidene-5,6-dihydro-4H-cyclopenta[b]thiophene-3-carbohydrazide (9). - To a solution of compound $7(2.53 \mathrm{~g}, 0.01 \mathrm{~mol})$ in 1,4-dioxane $(40 \mathrm{~mL})$ containing triethylamine $(0.50 \mathrm{~mL})$, elemental sulphur $(0.32 \mathrm{~g}, 0.01 \mathrm{~mol})$ was added. The reaction mixture was heated under reflux for $1 \mathrm{~h}$, then poured onto ice/water containing a few drops of hydrochloric acid and the formed solid product was collected by filtration.

2-Cyano-2-(2-cyclopentylidene)phenylacetohydrazide (10). - To a solution of compound $1(1.65 \mathrm{~g}, 0.01 \mathrm{~mol})$ in 1,4-dioxane $(40 \mathrm{~mL})$, acetophenone $(1.20 \mathrm{~g}, 0.01 \mathrm{~mol})$ was added. The reaction mixture was heated under reflux for $4 \mathrm{~h}$, then left to cool overnight and the formed solid product was collected by filtration.

2-Cyano-2-(2-(2-phenylhydrazono)cyclopentylidene)acetohydrazide (11). - To a cold solution $\left(0-5{ }^{\circ} \mathrm{C}\right)$ of compound $1(1.65 \mathrm{~g}, 0.01 \mathrm{~mol})$ in ethanol $(95 \%, 40 \mathrm{~mL})$ containing sodium acetate $(4.0 \mathrm{~g})$, benzenediazonium chloride $(0.01 \mathrm{~mol})$ [prepared by the addition of sodium nitrite solution $(0.70 \mathrm{~g}, 0.01 \mathrm{~mol}$ in $10 \mathrm{~mL}$ water) to a cold solution of aniline $(0.94 \mathrm{~g}, 0.01 \mathrm{~mol})$ in concentrated hydrochloric acid $(8.0 \mathrm{~mL})]$ was added dropwise under continuous stirring. The reaction mixture was stirred at room temperature for $2 \mathrm{~h}$ and the formed solid product was collected by filtration.

N'-(1-chloropropan-2-ylidene)-2-cyano-2-cyclopentylideneacetohydrazide (12). - To a solution of compound $1(1.65 \mathrm{~g}, 0.01 \mathrm{~mol})$ in 1,4-dioxane $(40 \mathrm{~mL}), \alpha$-chloroacetone $(0.92 \mathrm{~g}$, $0.01 \mathrm{~mol}$ ) was added. The reaction mixture was heated under reflux for $2 \mathrm{~h}$, then left to cool overnight and the formed solid product was collected by filtration.

2-Cyclopentylidene-3-(3,5-diamino-1H-pyrazol-1-yl)-3-oxopropanenitrile (13a) and 3-(3-amino-5-hydroxy-1H-pyrazol-1-yl)-2-cyclopentylidene-3-oxopropanenitrile (13b). General procedure. - To a solution of compound $\mathbf{1}(1.65 \mathrm{~g}, 0.01 \mathrm{~mol})$ in 1,4-dioxane $(40 \mathrm{~mL})$ containing triethylamine $(0.50 \mathrm{~mL})$, either malononitrile $(0.66 \mathrm{~g}, 0.01 \mathrm{~mol})$ or ethyl cyanoacetate $(1.13 \mathrm{~g}, 0.01 \mathrm{~mol})$ was added. The reaction mixture was heated under reflux for $2 \mathrm{~h}$, then poured onto ice/water containing a few drops of hydrochloric acid and the formed solid product was collected by filtration.

\section{Antitumor screening}

Fetal bovine serum (FBS) and L-glutamine were from Gibco Invitrogen Co. (UK). RPMI-1640 medium was from Cambrex (USA). Dimethyl sulfoxide (DMSO), doxorubicin, 
penicillin, streptomycin and sulforhodamine B (SRB) were from Sigma Chemical Co. (USA). Stock solutions of new compounds $\mathbf{1 - 1 3} \mathbf{3}, \mathbf{b}\left(25 \mu \mathrm{mol} \mathrm{L}{ }^{-1}\right)$ were prepared in DMSO and kept at $-20{ }^{\circ} \mathrm{C}$. Appropriate dilutions of the compounds were prepared just prior to the assays.

Cell cultures. - Three human tumor cell lines, MCF-7 (breast adenocarcinoma), NCI-H460 (non-small cell lung cancer), and SF-268 (CNS cancer) were used. MCF-7 was obtained from the European Collection of Cell Cultures (Salisbury, UK) and NCI-H460 and SF-268 were kindly provided by the National Cancer Institute (Cairo, Egypt). They grew as monolayers and were routinely maintained in RPMI-1640 medium supplemented with $5 \%$ heat inactivated FBS, $2 \mathrm{mmol} \mathrm{L}^{-1}$ glutamine and antibiotics (penicillin $100 \mu \mathrm{g} \mathrm{mL}^{-1}$, streptomycin $100 \mu \mathrm{g} \mathrm{mL}-1$ ), at $37{ }^{\circ} \mathrm{C}$ in a humidified atmosphere containing $5 \% \mathrm{CO}_{2}$. Exponentially growing cells were obtained by plating $1.5 \times 10^{5}$ cell mL $\mathrm{mL}^{-1}$ for both of MCF-7, SF-268 and $0.75 \times 10^{4}$ cell $\mathrm{mL}^{-1}$ for NCI-H460, followed by $24 \mathrm{~h}$ of incubation. The effect of the solvent (DMSO) on the growth of these cell lines was evaluated in all experiments by exposing untreated control cells to the maximum concentration $(0.5 \%)$ of DMSO used in each assay where doxorubicin was used as the reference compound.

Results are given in concentrations that were able to cause $50 \%$ cell growth inhibition $\left(G I_{50}\right)$ after a continuous exposure for $48 \mathrm{~h} \pm$ SEM of three-independent experiments performed in duplicate.

\section{RESULTS AND DISCUSSION}

\section{Chemistry}

The reaction of cyanoacetylhydrazine with cyclopentanone using ammonium acetate gave the 2-cyano-2-cyclopentylideneacetohydrazide (1). The structure elucidation of the latter product was based on analytical and spectral data. Thus, the ${ }^{1} \mathrm{H}$ NMR spectrum showed the presence of two multiplets at $\delta 1.17-2.34 \mathrm{ppm}$ corresponding to the four $\mathrm{CH}_{2}$ groups, a singlet at $\delta 3.83 \mathrm{ppm}$ corresponding to the $\mathrm{NH}_{2}$ group and a singlet $\left(\mathrm{D}_{2} \mathrm{O}\right.$ exchangeable) at $\delta 8.54 \mathrm{ppm}$ indicating the $\mathrm{NH}$ group. Moreover, its ${ }^{13} \mathrm{C}$ NMR spectrum showed the presence of signals at $\delta$ of $28.5,28.7,39.4,165.0$, indicating cyclopentyl C, a signal at $\delta 90.3$, equivalent to the quaternary $\mathrm{C}$ attached to the cyano group, a signal at $\delta 115.8$ confirming the $\mathrm{CN}$ group and a signal at $\delta 165.8$, corresponding to the $\mathrm{C}=\mathrm{O}$ goup. The reaction of compound $\mathbf{1}$ with elemental sulphur in the presence of triethylamine gave the 2-amino-5,6-dihydro- $4 \mathrm{H}$-cyclopenta[ $b]$ thiophene-3-carbohydrazide (2). The analytical and spectral data of the latter product were consistent with its proposed structure. Thus, the mass spectrum showed $m / z=197$ corresponding to the molecular ion peak. Moreover, the ${ }^{1} \mathrm{H}$ NMR spectrum showed two multiplets at $\delta 1.16-2.37$ ppm equivalent for the three $\mathrm{CH}_{2}$ groups, two singlets $\left(\mathrm{D}_{2} \mathrm{O}\right.$ exchangeable) at $\delta 3.80$ and $4.66 \mathrm{ppm}$ corresponding to the two $\mathrm{NH}_{2}$ and a singlet $\left(\mathrm{D}_{2} \mathrm{O}\right.$ exchangeable) at $\delta 8.77 \mathrm{ppm}$ indicting the $\mathrm{NH}$ group. The ${ }^{13} \mathrm{C}$ NMR spectrum showed, beside the expected signals for the cyclopentyl ring, signals at $\delta 118.3,133.6,139.4,145.2$, corresponding to the thiophene carbons. Compound 2 underwent ready diazotization in concentrated sulphuric acid at $0{ }^{\circ} \mathrm{C}$ and sodium nitrite solution to give the corresponding non isolable 2-diazosulphate salt. The latter coupled with either ethyl cyanoacetate or ethyl acetoacetate to give the 2-cyano-2-(3-(hydrazinecarbonyl)-5,6-dihydro-4H-cyclopenta[b]thiophen-2-yl)hy- 
drazono)-acetate (3) and the ethyl 2-(2-(3-(hydrazinecarbonyl)-5,6-dihydro- $4 H$-cyclopenta[b]thiophen-2-yl)hydrazono)-3-oxobutanoate (4), respectively. The mass spectra of both compounds showed molecular masses at $\mathrm{m} / \mathrm{z} 321$ and 338, respectively. The ${ }^{1} \mathrm{H}$ NMR spectrum of compound 3 revealed the presence of a triplet at $\delta 1.13 \mathrm{ppm}$ indicating the ester $\mathrm{CH}_{3}$ group, a quartet at $\delta 4.22 \mathrm{ppm}$ for the ester $\mathrm{CH}_{2}$ group and two singlets $\left(\mathrm{D}_{2} \mathrm{O}\right.$ exchangeable) at $\delta 8.81$ and $10.27 \mathrm{ppm}$ corresponding to the two NH groups. The reaction of compound 1 with phenylisothiocyanate gave the 2-(2-cyano-2-cyclopentylideneacetyl)- $N$-phenylhydrazinecarbothioamide (5). The latter compound underwent heterocyclization when reacting with either $\alpha$-chloroacetone or phenacylbromide to afford either the 2-cyano-2-cyclopentylidene- $N$ '-(4-methyl-3-phenylthiazol-2(3H)-ylidene)acetohydrazide (6a) or the 2-cyano-2-cyclopentylidene- $\mathrm{N}^{\prime}$-(3,4-diphenylthiazol-2(3H)-ylidene)acetohydrazide $(6 \mathbf{b})$, respectively.

Compound 1 was allowed to react with benzaldehyde under two different conditions to give two different products. Thus, carrying out the reaction in refluxing 1,4-dioxane afforded the $N^{\prime}$-benzylidene-2-cyano-2-cyclopentylideneacetohydrazide (7). On the other hand, carrying out the same reaction in the presence of a catalytic amount of piperidine gave the 2-(2-benzylidenecyclopentylidene)-2-cyanoacetohydrazide (8). The ${ }^{1} \mathrm{H}$ NMR spectra of compounds 7 and 8 showed signals at $\delta 6.88$ and $6.87 \mathrm{ppm}$, respectively corresponding to the $\mathrm{CH}=\mathrm{N}$ group present in 7 and the $\mathrm{CH}=\mathrm{C}$ present in 8 and multiplets at $\delta$ 7.30-7.38 and 7.29-7.35 ppm, respectively, indicating the phenyl group. The reaction of compound 7 with elemental sulphur in the presence of triethylamine afforded the 2-amino-5,6-dihydro- $4 \mathrm{H}$-cyclopenta[ $b]$ thiophene-3- $\mathrm{N}$-benzal-carbohydrazide (9). The ${ }^{13} \mathrm{C}$ NMR spectrum of the latter product showed, beside the expected signals for the cyclopentyl group, signals at $\delta$ at 130.1,132.8, 139.4, 147.3, indicating phenyl and thiophene $\mathrm{C}$, a signal at $\delta 165.3$, corresponding to the $\mathrm{C}=\mathrm{O}$ and a signal at $\delta 172.4$, indicating $\mathrm{C}=\mathrm{N}$.

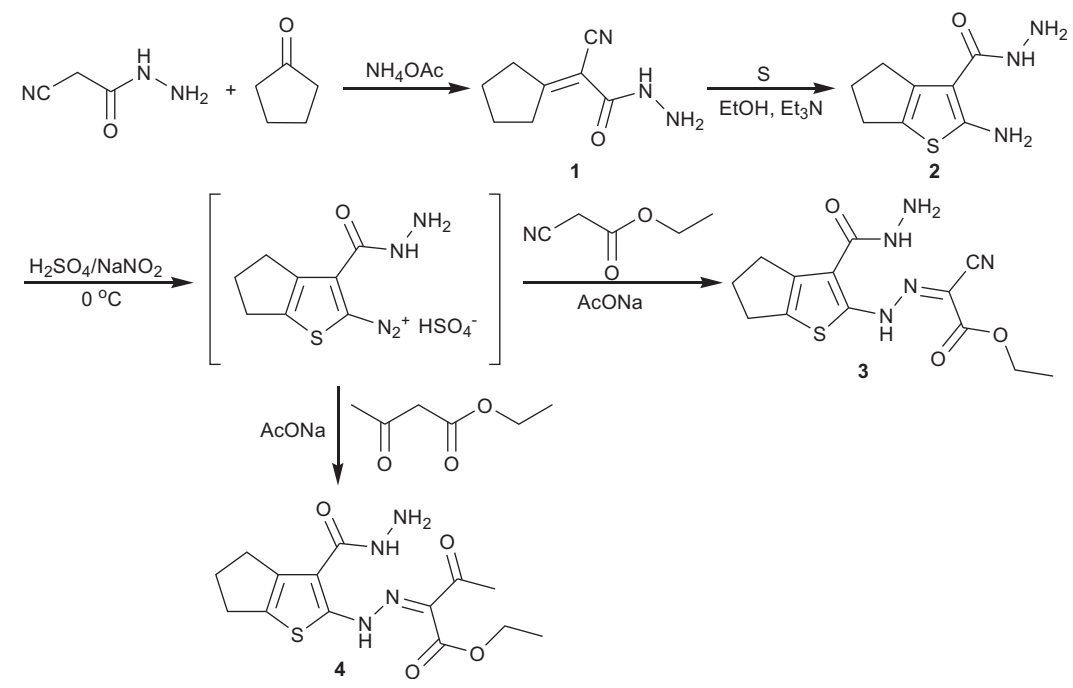

Scheme 1. 
W. W. Wardakhan et al:: Synthesis and anti-tumor evaluation of novel hydrazide and hydrazide-hydrazone derivatives, Acta Pharm. 63 (2013) 45-57.
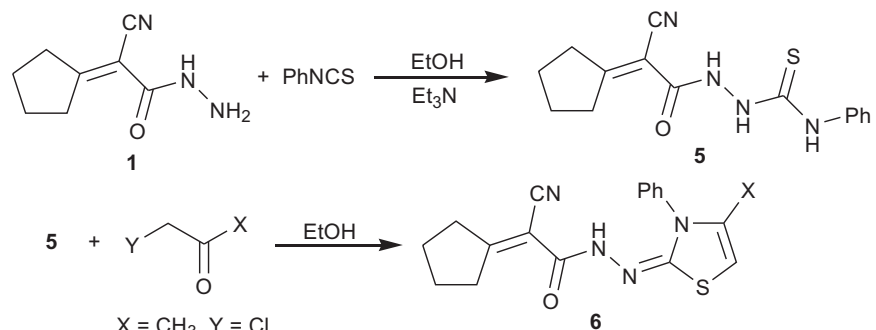

$\mathrm{X}=\mathrm{Ph}, \mathrm{Y}=\mathrm{Br} \quad \mathbf{a} \mathrm{X}=\mathrm{CH}_{3}, \mathbf{b} \quad \mathrm{X}=\mathrm{Ph}$

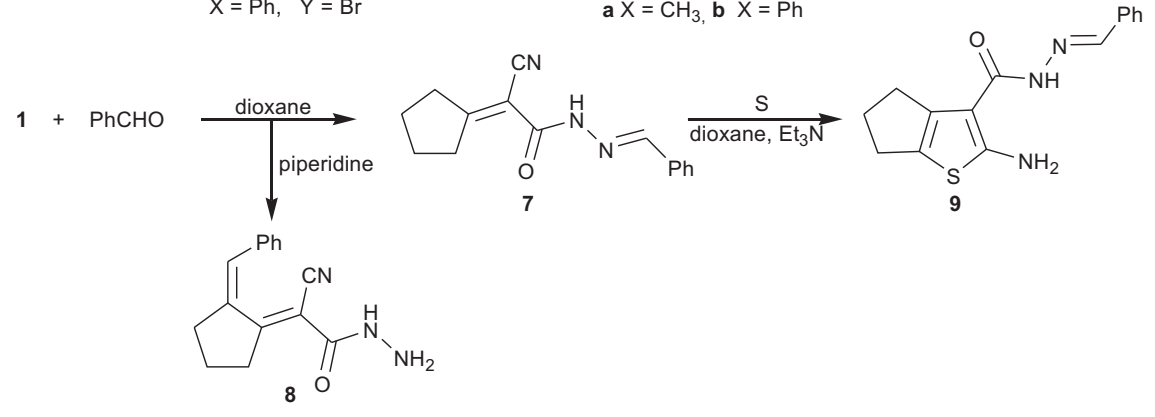

Scheme 2.

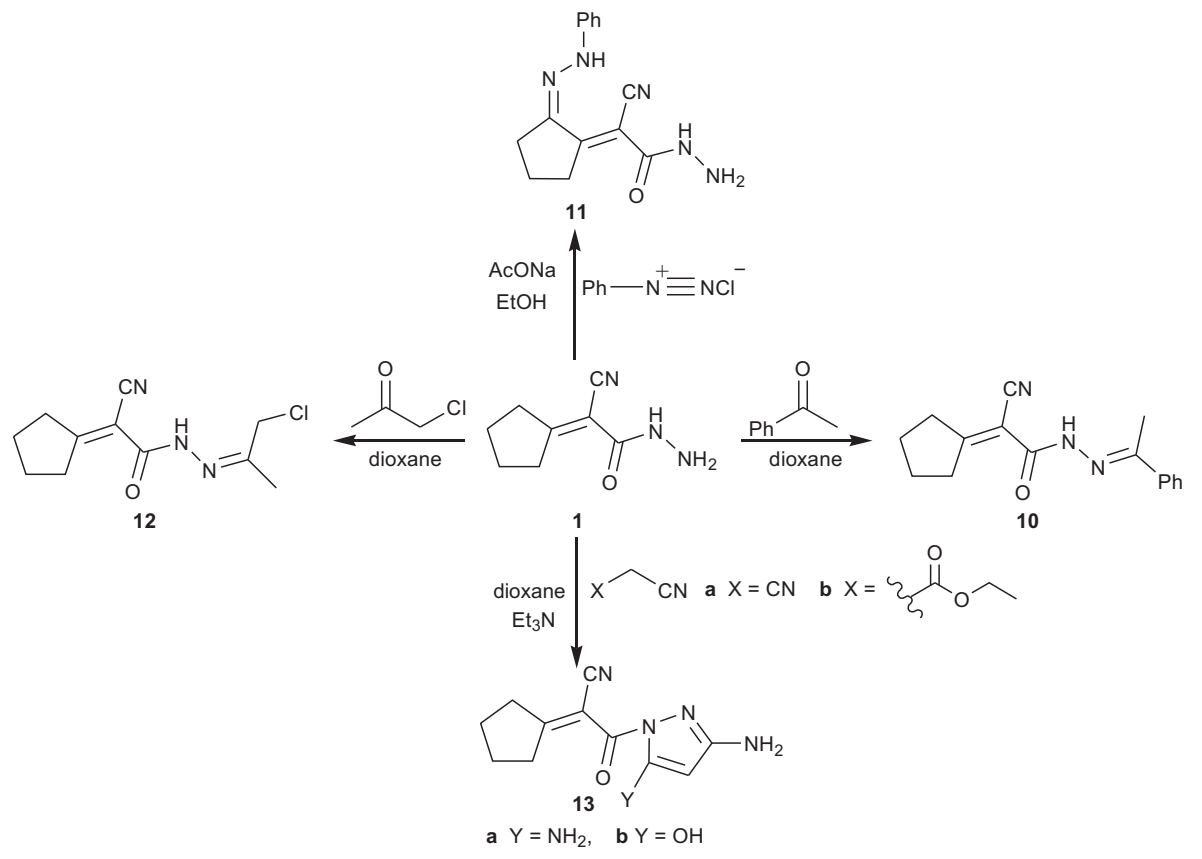

Scheme 3. 
The reaction of compound 1 with acetophenone in 1,4-dioxane gave the 2-cyano-2-(2-(2-cyclopentylidene)phenylacetohydrazide (10). Moreover, the reaction of compound 1 with benzenediazonium chloride in the presence of sodium acetate at $0{ }^{\circ} \mathrm{C}$ gave the 2-cyano-2-(2-(2-phenylhydrazono)cyclopentylidene)acetohydrazide (11). On the other hand, the reaction of 1 with chloroacetone gave the $N^{\prime}$-(1-chloropropan-2-ylidene)-2-cyano-2cyclopentylideneacetohydrazide (12). Finally, we moved towards studying the reactivity of compound 1 with cyanomethylene reagents with the aim of its heterocyclization to the potentially antitumor pyranoles. Thus, compound 1 reacted with either malononitrile or ethyl cyanoacetate to give the 2-cyclopentylidene-3-(3,5-disubstituted-1H-pyrazol-1-yl)-3-oxopropanenitriles 13a and 13b, respectively. The analytical and spectral data of $\mathbf{1 3} \mathbf{a}$ and $\mathbf{1 3 b}$ are in agreement with their respective structures.

\section{Antitumor activity and structure activity relationship}

The effect of compounds $\mathbf{1 - 1 3} \mathbf{a}, \mathbf{b}$ was evaluated through the in vitro growth of three human tumor cell lines representing different tumor types, namely, breast adenocarcinoma (MCF-7), non-small cell lung cancer (NCI-H460) and CNS cancer (SF-268), after continuous exposure for $48 \mathrm{~h}$. The results summarized in Table III showed that all the compounds were able to inhibit the growth of the human tumor cell lines in a dose-dependent manner. 2-Cyano-2-cyclophentylideneacetohydrazido- $N$-(4-cyano-3-phenyl-4-phenylthiazol-2-ylideno)-hydrazone (6b), 2-cyano-2-cyclopentylideneaceto- $N$-benzalhydrazide (7), 2-cyano-2-(2-(2-phenylhydrazono)cyclopentylidene)acetohydrazide (11) and 3-(3-amino-5-hydroxy-1H-pyrazol-1-yl)-2-cyclopentylidene-3-oxopropanenitrile (13b) showed the best results. It is

Table III. Effects of compounds 1-13a,b on the growth of three human tumor cell lines

\begin{tabular}{cccc}
\hline Compd. & \multicolumn{3}{c}{$G I_{50}(\mu \mathrm{mol} \mathrm{L}-1)$} \\
\cline { 2 - 4 } & MCF-7 & NCI-H460 & SF-268 \\
\hline $\mathbf{1}$ & $12.0 \pm 0.6$ & $10.4 \pm 2.4$ & $16.8 \pm 4.8$ \\
$\mathbf{2}$ & $18.0 \pm 4.2$ & $20.3 \pm 3.6$ & $26 \pm 2.8$ \\
$\mathbf{3}$ & $30.2 \pm 10.9$ & $22.7 \pm 2.8$ & $40.2 \pm 6.0$ \\
$\mathbf{4}$ & $8.2 \pm 1.9$ & $12.8 \pm 4.8$ & $8.0 \pm 2.6$ \\
$\mathbf{5}$ & $60.2 \pm 3.4$ & $44.7 \pm 6.1$ & $16.4 \pm 4.0$ \\
$\mathbf{6 a}$ & $10.6+1.2$ & $6.1 \pm 2.2$ & $2.0 \pm 1.2$ \\
$\mathbf{6 b}$ & $0.02 \pm 0.01$ & $0.08 \pm 0.01$ & $0.06 \pm 0.02$ \\
$\mathbf{7}$ & $2.2 \pm 0.8$ & $4.6 \pm 0.4$ & $1.2 \pm 0.8$ \\
$\mathbf{8}$ & $30.0 \pm 2.5$ & $22.0 \pm 4.6$ & $20.5 \pm 2.8$ \\
$\mathbf{9}$ & $10.0 \pm 0.8$ & $8.3 \pm 2.8$ & $16.5 \pm 4.0$ \\
$\mathbf{1 0}$ & $40.4 \pm 2.8$ & $22.1 \pm 0.6$ & $38.3 \pm 0.5$ \\
$\mathbf{1 1}$ & $0.01 \pm 0.008$ & $0.01 \pm 0.006$ & $0.08 \pm 0.08$ \\
$\mathbf{1 2}$ & $77.8 \pm 10.0$ & $64.2 \pm 8.4$ & $70.2 \pm 12.6$ \\
$\mathbf{1 3 a}$ & $14.2 \pm 8.2$ & $10.0 \pm 2.6$ & $10.2 \pm 4.8$ \\
$\mathbf{1 3 b}$ & $0.4 \pm 0.1$ & $0.2 \pm 0.01$ & $0.1 \pm 0.02$ \\
Doxorubicin & $0.04 \pm 0.008$ & $0.09 \pm 0.008$ & $0.09 \pm 0.007$ \\
\hline
\end{tabular}




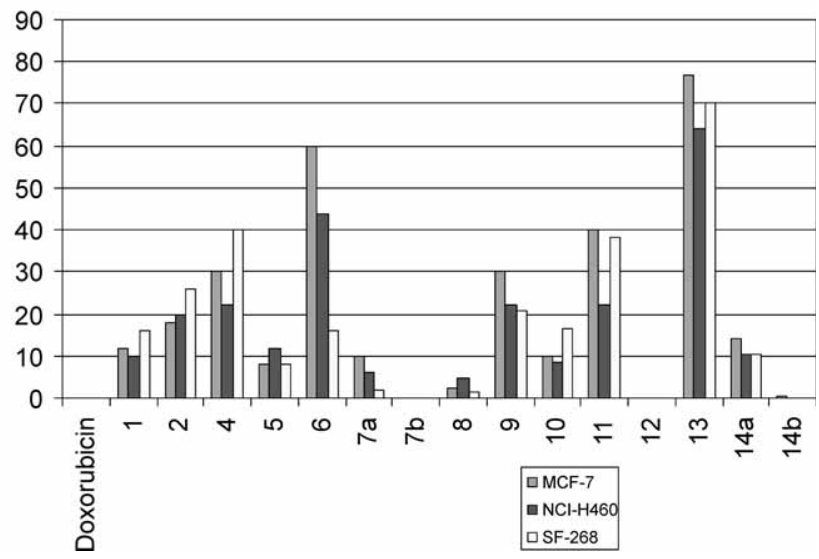

Fig. 1.

convenient to notice that compounds $\mathbf{6 b}$ and $\mathbf{1 1}$ have the highest cytotoxicity and their activities are higher than that of doxorubicin. Such high cytotoxicity of both compounds is attributed to the presence of the thiazole moiety bearing two phenyl groups in $\mathbf{6 b}$ and the high nitrogen content of the hydrazide and hydrazone moieties that are present in 11. On the other hand, comparing the cytotoxicity effect of compounds $\mathbf{6 a}$ and $\mathbf{6 b}$, one can say that the presence of the 3,4-diphenyl-thiazole moiety in $\mathbf{6 b}$ is responsible for its higher cytotoxicity effect than the 3-phenyl-4-methylthiazole moiety in 6a. On the other hand, compounds 4, 6a, 7, 9 and 13a showed moderate growth inhibitory effects. Comparing the activities of compounds $\mathbf{1 3 a}$ and $\mathbf{1 3 b}$, it is observed that compound $\mathbf{1 3 b}$ showed higher cytotoxicity compared to compound 13a. Higher cytotoxicity of $\mathbf{1 3 b}$ is due to the presence of the electron withdrawing 5-hydroxy group attached to the pyrazole ring while in case of compound 13a, the 3,5-diamino groups lowered its cytotoxicity. The alicyclic compounds 5, $\mathbf{1 0}$ and $\mathbf{1 2}$ showed the lowest inhibitory effect on the three cancer cell lines.

\section{CONCLUSIONS}

The above results allow the conclusion that administration of the tested compounds to breast adenocarcinoma (MCF-7), non-small cell lung cancer (NCI-H460) and CNS cancer (SF-268) cells showed promising anticancer activity. The most potent compounds were 2-cyano-2-cyclophentylideneacetohydrazido- $N$-(4-cyano-3-phenyl-4-phenyl-thiazol-2-ylideno) hydrazone (6b) and 2-cyano-2-(2-phenylhydrazono)-cyclopentylideneacetohydrazide (11) with inhibitory effects higher than that of the reference doxorubicin. 
W. W. Wardakhan et al.: Synthesis and anti-tumor evaluation of novel hydrazide and hydrazide-hydrazone derivatives, Acta Pharm. 63 (2013) 45-57.

\section{REFERENCES}

1. S. K. Bharti, G. Nath, R. Tilak and S. K. Singh, Synthesis, anti-bacterial and anti-fungal activities of some novel Schiff bases containing 2,4-disubstituted thiazole ring, Eur. J. Med. Chem. 45 (2010) 651-660; DOI: 10.1016/j.ejmech.2009.11.008.

2. C. Loncle, J. M. Brunel, N. Vidal, M. Dherbomez and Y. Letourneux, Synthesis and antifungal activity of cholesterol-hydrazone derivatives, Eur. J. Med. Chem. 39 (2004) 1067-1071; DOI: 10.1016/ j.ejmech.2004.07.005.

3. S. P. Garoufalias, N. Pouli, P. Marakos and A. C. Ladas, Synthesis, antimicrobial and antifungal activity of some new 3-substituted derivatives of 4-(2,4-dichlorophenyl)-5-adamantyl-1 H-1,2,4-triazole, Farmaco 57 (2002) 973-977; DOI: 10.1016/S0014-827X(02)01273-9.

4. P. Vicini, F. Zani, P. Cozzini and I. Doytchinova, Hydrazones of 1,2-benzisothiazole hydrazides: synthesis, antimicrobial activity and QSAR investigations, Eur. J. Med. Chem. 37 (2002) 553-567; DOI: 10.1016/S0223-5234(02)01378-8.

5. F. D. Popp, Potential anticonvulsant. XII. Anticonvulsant activity of some aldehyde derivatives, Eur. J. Med. Chem. 24 (1989) 313-316; DOI: 10.1016/0223-5234(89)90016-0.

6. S. K. Sridhar, S. N. Pandeya, J. P. Stables and R. Atmakuru, Anticonvulsant activity of hydrazones, Schiff and Mannich bases of isatin derivatives, Eur. J. Pharm. Sci. 16 (2002) 129-132; DOI: 10.1016/S0928-0987(02)00077-5.

7. S. G. Küçükgüzel, S. Rollas, I. Küçükgüzel and M. Kiraz, Synthesis and antimycobacterial activity of some coupling products from 4 -aminobenzoic acid hydrazones, Eur. J. Med. Chem. 34 (1999) 1093-1100; DOI: 10.1016/S0223-5234(99)00129-4.

8. B. K. Kaymakçýoğlu and S. Rollas, Synthesis, characterization and evaluation of antituberculosis activity of some hydrazones, Farmaco 57 (2002) 595-599; DOI: 10.1016/S0014-827X(02)01255-7.

9. S. G. Küçükgüzel, A. Mazi, F. Sahin, S. Öztürk and J. P. Stables, Synthesis and biological activities of diflunisal hydrazide-hydrazones, Eur. J. Med. Chem. 38 (2003) 1005-1013; DOI: 10.1016/ j.ejmech.2003.08.004.

10. A. R. Todeschini, A. L. P. de Miranda, K. C. M. da Silva, S. C. Parrini and E. Barreiro, Synthesis and evaluation of analgesic, antiinflammatory and antiplatelet properties of new 2-pyridylarylhydrazone derivatives, Eur. J. Med. Chem. 33 (1998) 189-199; DOI: 10.1016/S0223-5234(98)80008-1.

11. M. A. Gaston, L. R. S. Dias, A. C. C. Freitas, A. L. P. Miranda and E. J. Barreiro, Synthesis and analgesic properties of new 4-arylhydrazone $1 \mathrm{H}$ pyrazole[3,4-b]pyridine derivatives, Pharm. Acta Helv. 71 (1996) 213-219; DOI: 10.1016/0031-6865(96)00012-X.

12. P. Melnyk, V. Leroux, C. Sergheraert and P. Grellier, Design, synthesis and in vitro antimalarial activity of an acylhydrazone library, Bioorg. Med. Chem. Lett. 16 (2006) 31-35; DOI: 10.1016/j.bmcl. 2005.09.058.

13. N. Terzioglu and A. Gursoy, Synthesis and anticancer evaluation of some new hydrazone derivatives of 2,6-dimethylimidazo[2,1-b][1,3,4]thiadiazole-5-carbohydrazide, Eur. J. Med. Chem. 38 (2003) 781-786; DOI: 10.1002/chin.200401111.

14. S. M. Sondhi, M. Dinodia and A Kumar, Synthesis, anti-inflammatory and analgesic activity evaluation of some amidine and hydrazone derivatives, Bioorg. Med. Chem. 14 (2006) 4657-4663; DOI: 10.1016/j.bmc.2006.02.014.

15. C. Boga, L. Fiume, M. Baglioni, C. Bertucci, C. Farina, F. Kratz, M. Manerba, M. Naldi and G. Stefano, Characterisation of the conjugate of the (6-maleimidocaproyl)hydrazone derivative of doxorubicin with lactosaminated human albumin by ${ }^{13} \mathrm{C}$ NMR spectroscopy, Eur. J. Pharm. Sci. 38 (2009) 262-269; DOI: 10.1016/j.ejps.2009.08.001.

16. M. C. Garnett, Targeted drug conjugates: principles and progress, Adv. Drug Deliv. Rev. 53 (2001) 171-216; DOI: 10.1016/S0169-409X(01)00227-7. 
17. P. C. Rodrigues, K. Scheuermann, C. Stockmar, G. Maier, H. Fiebig, C. Unger, R. Mülhaupt and F. Kratz, Synthesis and in-vitro efficacy of acid-sensitive poly(ethylene glycol) paclitaxel conjugates, Bioorg. Med. Chem. Lett. 13 (2003) 355-360; DOI: 10.1016/S0960-894X(02)01002-8.

18. O. I. El-Sabbagh and H. M. Rady, Synthesis of new acridines and hydrazones derived from cyclic â-diketone for cytotoxic and antiviral evaluation, Eur. J. Med. Chem. 44 (2009) 3680-3686; DOI: 10.1002 /chin.200948062.

19. H. Krakovicova, T. Etrych and K. Ulbrich, HPMA-based polymer conjugates with drug combination, Eur. J. Pharm. Sci. 37 (2009) 405-412; DOI: 10.1016/j.ejps.2009.03.011.

20. H. Z. Zhang, J. Drewe, B. Tseng, S. Kasibhatla and S. X. Cai, Discovery and SAR of indole-2-carboxylic acid benzylidene-hydrazides as a new series of potent apoptosis inducers using a cell-based HTS assay, Bioorg. Med. Chem. 12 (2004) 3649-3655; DOI: 10.1016/j.bmc.2004.04.017.

21. P. Vicini, M. I. Incerti, P. L. Colla and R. Loddo, Anti-HIV evaluation of benzoŠdĆisothiazole hydrazones, Eur. J. Med. Chem. 44 (2009) 1801-1807; DOI: 10.1016/j.ejmech.2008.05.030.

22. V. M. Rahman, S. Mukhtar, W. H. Ansari and G. Lemiere, Synthesis, stereochemistry and biological activity of some novel long alkyl chain substituted thiazolidin-4-ones and thiazan-4-one from 10undecenoic acsid hydrazide, Eur. J. Med. Chem. 40 (2005) 173-184; DOI: 10.1016/j.ejmech.2004. 10.003 .

23. J. R. Dimmock, S. C. Vashishtha and J. P. Stables, Anticonvulsant properties of various acetylhydrazones, oxamoylhydrazones and semicarbazones derived from aromatic and unsaturated carbonyl compounds, Eur. J. Med. Chem. 35 (2000) 241-248; DOI: 10.1016/S0223-5234(00)00123-9.

24. D. Kaushik, S. A. Khan, G. Chawla and S. Kumar, N'-[5-chloro-3-methyl-1-phenyl-1H-pyrazol-4-yl)methylene]2/4-substituted hydrazide: Synthesis and anticonvulsant activity, Eur. J. Med. Chem. 45 (2010) 3943-3949; DOI: 10.1016/j.ejmech.2010.05.049.

25. S. G. Küçükgüzel, A. Mazi, F. Sahin, S. Öztürk and J. Stables, Synthesis and biological activities of diflunisal hydrazide-hydrazones, Eur. J. Med. Chem. 38 (2003) 1005-1013. DOI: 10.1016/ j.ejmech.2003.08.004.

26. Y. L. Xia, F. Chuan-Dong, B. X. Zhao, J. Zhao, D. S. Shin and J. Y. Miaom, Synthesis and structure-activity relationships of novel 1-arylmethyl-3-aryl-1H pyrazole-5-carbohydrazide hydrazone derivatives as potential agents against A549 lung cancer cells, Eur. J. Med. Chem. 43 (2008) 2347-2353; DOI: 10.1016/j.ejmech.2008.01.021.

27. C. Menendez, A. Chollet, F. Rodriguez, C. Inard, M. R. Pasca, C. Lherbet and M. Baltas, Chemical synthesis and biological evaluation of triazole derivatives as inhibitors of InhA and antituberculosis agents, Eur. J. Med. Chem. 52 (2012) 275-283; DOI: 10.1016/j.ejmech.2012.03.029.

28. N. Özbek, S. Alyar and N. Karacan, Experimental and theoretical studies on methanesulfonic acid 1-methylhydrazide: Antimicrobial activities of its sulfonyl hydrazone derivatives, J. Mol. Struct. 938 (2009) 48-53; DOI: 10.1016/j.molstruc.2009.09.002.

29. R. M. Mohareb and J. Schatz, Anti-tumor and anti-leishmanial evaluations of 1,3,4-oxadiazine, pyran derivatives derived from cross-coupling reactions of b-bromo- $6 \mathrm{H}-1,3,4$-oxadiazine derivatives, Bioorg. Med. Chem. 19 (2011) 2707-2713; DOI: 10.1016/j.bmc.2011.02.051. 